\title{
Transition of a Judo Program from In-Person to Remote Delivery During COVID-19 for Youth with Autism Spectrum Disorder
}

\author{
Jeanette M. Garcia ${ }^{1}$ (D) - Braden S. Cathy ${ }^{1} \cdot$ Ana V. Garcia $^{2} \cdot$ Riley Shurack $^{1} \cdot$ Keith Brazendale $^{1} \cdot$ Nicholas Leahy $^{3}$. \\ David Fukuda $^{3}$. Shawn Lawrence ${ }^{4}$
}

Accepted: 13 February 2021 / Published online: 6 March 2021

(C) The Author(s), under exclusive licence to Springer Nature Switzerland AG part of Springer Nature 2021

\begin{abstract}
Objectives To examine the feasibility of a remote judo program for high school students with autism spectrum disorder (ASD) during the COVID-19 pandemic.

Methods Nine high school students with ASD had been participating in an in-person judo program that transitioned to a livestream format once the stay-at-home orders were mandated. The students completed surveys regarding their experience with the remote sessions. Their classroom teacher completed a semi-structured interview regarding the advantages and disadvantages of the remote format.

Results Eight out of the nine students attended $92 \%$ of the remote classes. All nine students reported feeling satisfied with the remote judo sessions. Reported benefits of the remote format included the structured routine during the pandemic and the opportunity for physical activity. Disadvantages included a lack of space and lack of one-on-one instruction.

Conclusions The remote judo program appeared to be both feasible and acceptable for youth with ASD.
\end{abstract}

Keywords Coronavirus $\cdot$ Autism $\cdot$ Adolescents $\cdot$ Physical activity $\cdot$ Online

There is growing interest in the benefits of physical activity (PA) for youth diagnosed with autism spectrum disorder (ASD) (Bremer et al. 2016; Healy et al. 2018). Prior studies have found that participation in PA may improve language and social skills, decrease negative behaviors, and lessen feelings of anxiety in this population (Bremer et al. 2016). Recent research has shown that youth with ASD may be drawn to activities, such as martial arts, due to the structure of exercises and the mind-body interaction (Bell et al. 2016; Garcia et al.

Jeanette M. Garcia

Jeanette.garcia@ucf.edu

1 Department of Health Sciences, College of Health Professions and Sciences, University of Central Florida, Orlando, FL 32816, USA

2 Department of Psychology, College of Sciences, University of Central Florida, Orlando, FL 32816, USA

3 School of Kinesiology and Physical Therapy, College of Health Professions and Sciences, University of Central Florida, Orlando, FL 32816, USA

4 School of Social Work, College of Community Innovation, and Education, University of Central Florida, Orlando, FL 32816, USA
2020). Judo, in particular, may be especially beneficial for youth with ASD, as it emphasizes mindfulness, improves balance and coordination, and focuses on self-defense and learning to fall safely (Garcia et al. 2020; Rivera et al. 2020). A recent study by Rivera et al. (2020) reported that youth with ASD demonstrated increased confidence and improved sociability after attending an 8-week judo program. Given the potential benefits of PA, especially PA that incorporates a mindbody connection, it is imperative that such opportunities be available to youth with ASD.

In March of 2020, the World Health Organization (WHO) classified the novel coronavirus (COVID-19) as a pandemic (Yarimkaya and Esenturk 2020). As a result, the majority of the USA mandated a stay-at-home order, resulting in the cancelation of all classes and after-school activities (Esenturk 2020). This disruption to routine may be especially upsetting for youth with ASD who have a tendency to prefer a structured schedule (Brazendale et al. 2020). This disruption, coupled with the unknown direction of the pandemic and the greater potential for self-isolation, may have adverse effects on physical and psychosocial heath for youth with ASD (Narzisi 2020). While PA and exercise may be an effective way to cope with the current situation, one of the barriers often 
cited by parents of children with ASD is the lack of opportunities to participate in structured PA programs, which would be further exacerbated by the stay-at-home order (Moore et al. 2020).

The use of remote instruction to provide PA programs, such as martial arts, would be a possible solution to promote PA for youth with ASD during the COVID-19 outbreak. Although the use of video-directed instruction has been found to be an evidenced-based treatment for effective learning in youth with ASD, there is limited research on the use of online or remote delivery of PA programs in this population (Sam et al. 2020). Given the importance of PA in this population, coupled with the possible negative effects of the COVID-19 outbreak, it is imperative to examine the feasibility and acceptability of an online PA program, particularly an activity such as judo, that may increase PA and improve psychosocial health in youth with ASD. Therefore, the purpose of this study was to examine the feasibility of a transition from an in-person judo program to remote sessions as a result of the COVID-19 pandemic in adolescents with ASD.

\section{Methods}

\section{Participants}

The current study was adapted from an existing in-person judo program for adolescents with ASD. Nine adolescents (89\% male; $16.87 \pm 1.36$ years; $78 \%$ White) with ASD were currently participating in the in-person judo program. Seven participants $(78 \%)$ were diagnosed with a co-occurring health condition. The most common conditions reported included attention deficit hyperactivity disorder, sensory disorders, and mood/anxiety disorders. Slightly over half of the sample (56\%) was on medication for ASD and/or another cooccurring health condition. The in-person program took place at a private school, located in Central Florida, that enrolled only children and adolescents with a primary diagnosis of ASD by a physician. All procedures for both the in-person and remote judo sessions were approved by the Institutional Review Board (IRB) at the investigators' university, and parental consent/child assent was given prior to any procedures.

\section{Procedures}

\section{In-Person Judo Sessions}

The in-person judo class consists of a convenience sample of nine high school students who were given the opportunity to participate in a judo class rather than their regular physical education program. Each student received a judo gi (uniform) during the second week of class. Judo sessions consisted of a warm-up, aerobic activity, meditative breathing, and a mixture of individual-centered and partner-centered exercises. Typically, these exercises included falling safely, ground-based movements, and standing-based throwing techniques. All sessions ended with meditative breathing and cooldown. Originally, the in-person program was scheduled to run twice a week for $45 \mathrm{~min}$ over a period of 12 weeks; however, the COVID-19 pandemic occurred after the 7th week of the study.

\section{Remote Judo Sessions}

Following a 2-week preparation period, the program was transitioned online and delivered remotely. All students were briefed on the format of the remote sessions and were instructed to wear comfortable clothes for the first week of remote judo. Parents of students were then able to pick up their judo gi at school during the second week. The classroom teacher provided all students, the judo instructor, and research assistants with the Zoom link to log in prior to the start of the class. Although the original in-person program had been designed for a 12-week period, it was decided that the remote sessions would continue for the remaining duration of the semester (6.5 weeks). Thus, the entire judo program ran for a total of 13.5 weeks with an additional 2-week transition period in-between the two delivery formats. Table 1 describes the key differences between the in-person and remote judo sessions.

\section{Measures}

\section{Participant Characteristics and Attendance}

Prior to the start of the in-person judo sessions, parents of students were asked to complete demographic surveys on behalf of their child. For both the in-person and remote judo sessions, a research assistant recorded the presence of all students at the start of the class. The homeroom teacher would notify the research assistant whether a student would be absent from that day's judo session, and provided a reason for the absence.

\section{Participant Surveys}

Following the end of the program, students were asked to complete a survey adapted from Porter et al. (2014) to examine student reaction to in-person vs. remote sessions. The survey consisted of four multiple choice questions that asked students to rate their experience with both the in-person and remote sessions (e.g., "In-person/remote sessions helped me learn"). Response options were provided on a 5-point Likert scale (Strongly agree, Agree, Neutral, Disagree, Strongly Disagree). Finally, there were four open-response questions 
Table 1 Characteristics of inperson and remote judo sessions

\begin{tabular}{ll}
\hline In-person judo sessions & Remote judo session \\
\hline Mix of standing and ground-based exercises & Primarily standing exercises \\
Mix of individual and partner centered exercises & Individual exercise \\
Focus on falling safely onto mats & Increased aerobic activity \\
Greater movement around gym & Focus on judo technique \\
Mindfulness exercises and deep breathing & Primarily stationary \\
Includes strength training and flexibility & Mindfulness exercises and deep breathing \\
\hline
\end{tabular}

that asked students about the advantages/disadvantages of the in-person and remote sessions.

\section{Teacher Interview}

A semi-structured interview was administered to the classroom teacher who supervised the students throughout their remote classes, and attended all of the in-person and remote judo sessions. Questions focused on the following topics: (1) student reaction to both the in-person and remote judo formats; (2) benefits and limitations to the remote format; and (3) suggestions for improvement of remote judo sessions. The interview took place via Zoom, and lasted approximately 45$50 \mathrm{~min}$. Interview content was later transcribed verbatim.

\section{Data Analyses}

Descriptive statistics were calculated for student demographic variables, session attendance rates, and survey response frequencies. Student survey responses from the 5-point Likert scale were further dichotomized into "Agree" or "Disagree/ Neutral," and a Chi-square test was conducted to examine any differences between the in-person and remote delivery formats. All quantitative analyses were conducted in SAS 9.4.

To analyze the qualitative data, two research assistants independently reviewed both the student open-ended responses and the teacher interview. The research assistants compiled the responses from the open-ended surveys, and organized the responses into "Likes" and "Dislikes" for both the inperson and remote-based programs. The research assistants then examined the teacher interview transcripts for pertinent themes, which were then summarized into tables.

\section{Results}

\section{Participant Attendance}

All nine students continued to participate in the judo program following the transition to Zoom sessions. For the in-person sessions, full attendance was noted for $4(29 \%)$ of the 14 possible classes. Only $3(21 \%)$ of the possible 14 sessions had 2 or more students absent. For the remote sessions, full attendance was noted for $4(31 \%)$ of the 13 possible classes. Several students were still adjusting to the Zoom format over the first 2 weeks, which resulted in $5(38 \%)$ of the 13 sessions missing 2 or more students. When excluding students who had technical difficulties during the first 2 weeks of the new format (4 sessions), only 1 class (12\%) was missing 2 or more students, and 8 of the 9 students attended $92 \%$ of the remote classes.

\section{Student Surveys}

There were no significant differences between the two formats for any of the items; however, the percentage of students who agreed with positive statements was slightly higher for inperson sessions compared to the remote sessions. Table 2 displays the results from the student surveys.

\section{Student Open-Ended Responses}

All nine students completed the open-response questions regarding their experience with both the in-person and Zoom judo formats. According to the open-ended response questions, seven of the nine students preferred the in-person sessions; however, all nine students reported satisfaction with the remote judo sessions since they were able to continue the program during the COVID-19 pandemic. The two students who preferred the remote sessions reported that they felt more comfortable, safer, and less self-conscious at home compared to the in-person sessions.

The primary disadvantages of the remote sessions included difficulty following the instructor on the video, a lack of hands-on assistance from the instructor, and the inability to practice partner-centered exercises. Students also mentioned that there were occasional disruptions to the video feed, which was distracting. Table 3 provides a summary of the student responses from the open-ended survey questions.

\section{Teacher Interview}

Results from the teacher interview were categorized into four themes: (1) benefits of remote judo during the quarantine; (2) 
Table 2 Student survey responses $(n=9)$

\begin{tabular}{lll}
\hline Statement & In-person & Remote (Zoom) \\
\hline Sessions were effective & $8(89 \%)^{1}$ & $6(67 \%)$ \\
Sessions helped me learn & $8(89 \%)$ & $7(78 \%)$ \\
Enjoyed the class using this specific format (in-person or Zoom) & $6(67 \%)$ & $4(44 \%)$ \\
Interested in taking a similar class using this format in the future & $4(44 \%)$ & $3(33 \%)$ \\
\hline
\end{tabular}

*No statistical significance between the two formats $(p>0.05)$

${ }^{1}$ All responses represent the $n$ and \% of students who "Agree" with each statement disadvantages of remote judo; (3) student preference for judo format; and (4) future suggestions for the use of remote sessions. Table 4 presents the various quotes that made up each theme.

Benefits of Remote Judo During the Quarantine Overall, the classroom teacher felt that the students benefitted from both judo formats; however, the teacher mentioned several specific benefits that the students experienced during the COVID-19 pandemic. A few of the benefits included providing structure during a time where their routine had been disrupted, and providing them with opportunities to be physically active, as the situation was not conducive to physical activity. A surprising benefit of remote judo was the parent involvement. Parents became more involved in the judo sessions as they were able to observe their child participating in the various exercises. The teacher even mentioned that several parents inquired about the sessions, and were pleased that their children were able to participate in a structured program.

Disadvantages of Remote Judo The teacher also reported a few disadvantages she observed during the remote sessions. First, one major disadvantage the teacher reported was the lack of space for practicing judo in the home. In fact, for the first week, students tested out different spaces to find the most suitable area for the judo exercises. Second, the classroom teacher reported that some students had technological difficulties with setting up the remote format; however, after the second week of remote judo, none of the students had any further difficulties.

Student Preference on Judo Format Similar to the student open responses, the classroom teacher felt that the majority of the students preferred the in-person sessions compared to the Zoom sessions. The teacher, however, pointed out that a few of the students preferred the remote judo sessions to a greater extent compared to the in-person sessions. The teacher suggested that the students who had higher levels of anxiety and were less active than the others appeared to prefer the remote format. The teacher also mentioned that, although the preference appeared to be for the in-person format, all the students enjoyed having the remote format, especially during the quarantine.

\section{Discussion}

The aim of this study was to evaluate a transition from an inperson judo session to remote judo program during the COVID-19 pandemic in a sample of adolescents with ASD. Findings from this study indicate that while the majority of the

Table 3 Student open-ended responses

\begin{tabular}{lllll}
\hline In-person judo sessions & & & Remote judo sessions & Dislike \\
\cline { 1 - 2 } \cline { 5 - 6 } Like & Dislike & & Like & No assistance \\
Hands-on & Other students could be distracting & & More comfortable at home & Less interaction \\
& & & Less distraction & Having to download zoom \\
Easy to get assistance & Self-conscious & Gym was too warm & Less self-conscious & Sweatier \\
Interacting with friends & Pressure to be good & Air-conditioned house & Hard to follow instructor techniques \\
& Anxious in group setting & & Could exercise during pandemic & Technical issues \\
& Fear of getting hurt & & Not isolated during quarantine & \\
\hline
\end{tabular}

*Two students did not have any "dislikes" regarding the in-person sessions 
Table 4 Teacher interview responses

\begin{tabular}{ll}
\hline Themes & Examples of relevant quotes \\
\hline Benefits of remote judo & "It [judo] gave them a sense of structure \& normalcy which they didn't have. \\
They were also anxious \& uncertain as a lot of them knew that the epidemic \\
was serious, but weren't quite sure what was going on. I noticed that once the \\
judo sessions started, they opened up more to me about their feelings \\
towards the pandemic and really anything else going on" \\
"A big thing with the in-person sessions is that they get that social interaction. \\
They interact with sensei and they interact with each other. Of course, not all \\
of the students are comfortable with social interaction and they may prefer \\
the Zoom sessions. One of the negative things is space at home. Also, they \\
don't have the [judo] mats so they can’t practice falling or do much floor \\
exercises." \\
"I think overall the in-person sessions will be most beneficial for social \\
interaction, although kids with autism who have more social anxiety may do \\
best with the Zoom sessions, at least at first. I think having the option for \\
both is good. I like that with Zoom, the kids can still interact with one \\
another." \\
"Maybe build their confidence up a bit through online judo and then advance to \\
in-person."
\end{tabular}

students preferred the in-person sessions, the remote sessions were an acceptable and safe alternative during the quarantine.

Although the majority of the students appeared to prefer the in-person sessions, it was noted that the students welcomed the opportunity to participate in the remote judo sessions, especially as it provided them structure and a familiar routine. The benefits of the structured routine are further supported by previous literature, which states that youth with ASD participate in healthier behaviors during structured days compared to non-structured days (Brazendale et al. 2020). Although anxiety was not directly measured in this study, the classroom teacher felt there was an acute effect from the program that eased their anxiety and encouraged more conversation immediately following the remote judo session. It is imperative to further investigate the effects of mind-body PA on anxiety in youth with ASD, as this could be potentially beneficial in times of prolonged stress and uncertainty. Finally, an unanticipated benefit of the remote judo program was the increased parent involvement. Prior research suggests that parent support is crucial for children with ASD; however, few studies have examined the relationship between family support and PA (Brown et al. 2020; Gregor et al. 2018). The inclusion of a livestream or video format that can take place at home may provide the opportunity for increased family involvement.

This study demonstrates the ability of an in-person judo program for adolescents with ASD to transition to a remote format during the COVID-19 pandemic. A recent study found that neurotypical adolescents decreased their physical activity during the mandatory stay-at-home order due to the pandemic (Moore 2020). The decrease in PA is concerning as exercise is beneficial for both physical and psychosocial health (Chen et al. 2020). For adolescents with ASD, this concern is even greater, as this population may derive even greater benefits from staying active, and participation in exercise that integrates mindfulness with aerobic activity may help to attenuate the levels of distress they may experience due to the disruption in their typical routine (Esenturk 2020; Rivera et al. 2020). The transition from their scheduled in-person judo class to remote instruction may be beneficial as it (1) promotes continued participation in PA; (2) includes meditative exercises, along with aerobic activity; and (3) encourages the students to adhere to part of their previous schedule as sessions were held at the same time and day as the in-person sessions.

\section{Limitations and Future Directions}

There are several limitations that should be noted in this pilot study. Firstly, the sample size is small which limits generalizability of the study. Secondly, as a result of the COVID-19 pandemic, we were unable to perform any objective measures for this study (e.g., accelerometers to assess physical activity during a judo session). Finally, although students reported their preferences for the in-person and remote formats, such findings should be reported with caution, as this study was designed as a result of the pandemic, and a true comparison between formats was not possible.

The current study has several implications. First, although in-person instruction may be preferred, the remote physical activity program appears to be an acceptable substitute in times where in-person sessions cannot occur. Second, although more research is necessary, a remote-based physical activity program may be a feasible starting point for youth with more severe ASD symptoms, such as greater levels of social anxiety, or youth who are more self-conscious and are new to exercise. Finally, results from the teacher interview 
suggest that similar remote-based programs may benefit from parental involvement and support.

Overall, the transition from in-person to remote judo sessions was well-accepted by this sample of high school students with ASD during the COVID-19 pandemic. Future research should also investigate the use of remote programs for individuals with more severe ASD symptoms, and whether other types of physical activity (e.g., strength training), besides judo, would still have similar positive effects.

Author Contribution JMG designed and executed the study, and wrote the paper. BSC collaborated in the execution of the study, data collection, and writing of the paper. AG and RS assisted with the analysis of the data and editing of the manuscript. KB collaborated with the execution of the study, and editing of the manuscript. NL, DF, and SL assisted with the writing and editing of the final manuscript.

\section{Declarations}

All procedures were approved by the University of Central Florida Institutional Review Board (IRB).

Research Involving Human Participants All procedures performed in studies involving human participants were in accordance with the ethical standards of the institutional and/or national research committee and with the 1964 Helsinki declaration and its later amendments or comparable ethical standards. No research was performed on animals in this study.

Informed Consent Informed consent was obtained from all individual participants included in the study.

Conflict of Interest The authors declare no competing interests.

\section{References}

Bell, A., Palace, K., Allen, M., \& Nelson, R. (2016). Using martial arts to address social and behavioral functioning in children and adolescents with autism spectrum disorder. Therapeutic Recreation Journal, 50(2). https://doi.org/10.18666/TRJ-2016-V50-I2-7287.

Brazendale, K., Brazendale, A. B., Garcia, J. M., Monroe, C. M., Weaver, R. G., \& Beets, M. W. (2020). Brief report: Obesogenic behaviors of children with developmental disabilities during summer. Journal of Autism and Developmental Disorders, 51, 734-740. https://doi.org/ 10.1007/s10803-020-04566-5.

Bremer, E., Crozier, M., \& Lloyd, M. (2016). A systematic review of the behavioural outcomes following exercise interventions for children and youth with autism spectrum disorder. Autism, 20(8), 899-915. https://doi.org/10.1177/1362361315616002.

Brown, D. M., Arbour-Nicitopoulos, K. P., Martin Ginis, K. A., LatimerCheung, A. E., \& Bassett-Gunter, R. L. (2020). Examining the relationship between parent physical activity and physical activity among children and youth with autism spectrum disorder. Autism, 24(7), 1783-1789. https://doi.org/10.1177/1362361320922658.

Chen, P., Mao, L., Nassie, G. P., Harmer, P., Ainsworth, B. E., \& Li, F. (2020). Coronavirus disease (COVID-19): The need to maintain regular physical activity while taking precautions. Journal of Sport and Health Science, 9(2), 103-104. https://doi.org/10.1016/j.jshs. 2020.02.001.

Esenturk, O. K. (2020). Parents' perceptions on physical activity for their children with autism spectrum disorders during the novel Coronavirus outbreak. International Journal of Developmental Disabilities. https://doi.org/10.1080/20473869.2020.1769333.

Garcia, J. M., Rivera, P., Renziehausen, J., Leahy, N., Fukuda, D., Samuels, J., \& Stout, J. (2020). Brief report: Preliminary efficacy of a judo program to promote participation in physical activity in youth with autism spectrum disorder. Journal of Autism and Developmental Disorders, 15, 1418-1424. https://doi.org/10.1007/ s10803-019-04338-w.

Gregor, S., Bruni, B., Grkinic, P., Schwartz, L., McDonald, A., Thile, P., Gabison, S., Gibson, B. E., \& Jachyra, P. (2018). Parents' perspectives of physical activity participation among Canadian adolescents with Autism Spectrum Disorders. Research in Autism Spectrum Disorders, 48, 53-62. https://doi.org/10.1016/j.rasd.2018.01.007.

Healy, S., Nacario, A., Braithwaite, R., \& Hoppe, C. (2018). The effect of physical activity interventions on youth with autism spectrum disorder: A meta-analysis. Autism Research, 11, 818-833. https://doi. org/10.1002/aur.1955.

Moore, S. A., Faulkner, G., Rhodes, R. E., Brussoni, M., Chulak-Bozzer, T., Ferguson, L. J., Mitra, R., O’Reilly, N., Spence, J. C., Vanderloo, L. M., \& Tremblay, M. S. (2020). Impact of the COVID-19 virus outbreak on movement and play behaviours of Canadian children and youth: A national survey. International Journal of Behavioral Nutrition and Physical Activity, 17, 85. https://doi.org/10.1186/ s12966-020-00987-8.

Narzisi, A. (2020). Handle the autism spectrum condition during Coronavirus (COVID-19) stay at home period: Ten tips for helping parents and caregivers of young children. Brain Sciences, 10, 207. https://doi.org/10.3390/brainsci10040207.

Porter, A. L., Pitterle, M. E., \& Hayney, M. S. (2014). Comparison of online versus classroom delivery of an immunization elective course. American Journal of Pharmaceutical Education, 78, 5. https://doi.org/10.5688/ajpe78596.

Rivera, P., Renziehausen, J., \& Garcia, J. M. (2020). Effects of an 8-week Judo program on behaviors in children with Autism Spectrum Disorder: A mixed-methods approach. Child Psychiatry \& Human Development. https://doi.org/10.1007/s10578-020-00994-7.

Sam, A., Cox, A., Savage, M. N., Waters, V., \& Odom, S. (2020). Disseminating information on evidence-based practices for children and youth with autism spectrum disorder: AFIRM. Journal of Autism and Developmental Disorders, 50. https://doi.org/10.1007/ s10803-019-03945-x.

Yarimkaya, E., \& Esenturk, O. K. (2020). Promoting physical activity for children with autism spectrum disorders during Coronavirus outbreak: Benefits, strategies, and examples. International Journal of Developmental Disabilities. https://doi.org/10.1080/20473869. 2020.1756115 .

Publisher's Note Springer Nature remains neutral with regard to jurisdictional claims in published maps and institutional affiliations. 